\title{
ANALYSIS OF SCHRÖDINGER MEANS
}

\author{
Per Sjölin and Jan-Olov Strömberg \\ KTH Royal Institute of Technology, Department of Mathematics \\ S-100 44 Stockholm, Sweden; persj@kth.se \\ KTH Royal Institute of Technology, Department of Mathematics \\ S-100 44 Stockholm, Sweden; jostromb@kth.se
}

Abstract. We study integral estimates of maximal functions for Schrödinger means.

\section{Introduction}

We shall study Schrödinger means $S_{t} f(x)$ defined by

$$
S_{t} f(x)=(2 \pi)^{-n} \int_{\mathbf{R}^{n}} \mathrm{e}^{i \xi \cdot x} \mathrm{e}^{i t|\xi|^{a}} \hat{f}(\xi) d \xi, \quad x \in \mathbf{R}^{n}, t \geq 0 .
$$

Here $f \in L^{2}\left(\mathbf{R}^{n}\right), n \geq 1, a>0$, and $\hat{f}$ denotes the Fourier transform $f$, defined by

$$
\hat{f}(\xi)=\int_{\mathbf{R}^{n}} \mathrm{e}^{-i \xi \cdot x} f(x) d x, \quad \xi \in \mathbf{R}^{n} .
$$

We shall use Sobolev spaces $H_{s}=H_{s}\left(\mathbf{R}^{n}\right), s \in \mathbf{R}$, with the norm given by

$$
\|f\|_{H_{s}}=\left(\int_{\mathbf{R}^{n}}\left(1+|\xi|^{2}\right)^{s}|\hat{f}(\xi)|^{2} d \xi\right)^{1 / 2} .
$$

Let $E$ denote a bounded set in $\mathbf{R}$. For $r>0$ we let $N_{E}(r)$ denote the minimal number $N$ of intervals $I_{l}, l=1,2, \ldots N$, of length $r$ such that $E \subset \bigcup_{1}^{N} I_{l}$. For $f$ belonging to the Schwartz class $\mathscr{S}$ we introduce the maximal function

$$
S_{E}^{*} f(x)=\sup _{t \in E}\left|S_{t} f(x)\right|, \quad x \in \mathbf{R}^{n} .
$$

In Sjölin and Strömberg [4] we proved the following theorem.

Theorem A. Assume that $n \geq 1$ and $a>0$ and $s>0$. If $f \in \mathscr{S}$, then one has

$$
\int_{\mathbf{R}^{n}}\left|S_{E}^{*} f(x)\right|^{2} d x \lesssim\left(\sum_{m=0}^{\infty} N_{E}\left(2^{-m}\right) 2^{-2 m s / a}\right)\|f\|_{H_{s}}^{2} .
$$

Here we write $A \lesssim B$ if there is a constant $C$ such that $A \leq C B$. In the case $E=[0,1]$ it is easy to see that Theorem A implies the estimate

$$
\left\|S_{E}^{*} f\right\|_{2} \lesssim\|f\|_{H_{s}}
$$

if $s>a / 2$.

Let $\left(t_{k}\right)_{1}^{\infty}$ be a sequence satisfying

$$
1>t_{1}>t_{2}>t_{3}>\cdots>0 \text { and } \lim _{k \rightarrow \infty} t_{k}=0 .
$$

Set $A_{j}=\left\{t_{k} ; 2^{-j-1}<t_{k} \leq 2^{-j}\right\}$ for $j=1,2,3, \ldots$ Let $\# A$ denote the number of elements in a set $A$. In [4] we used Theorem $\mathrm{A}$ to obtain the following results.

https://doi.org/10.5186/aasfm.2021.4616

2020 Mathematics Subject Classification: Primary 42B99.

Key words: Schrödinger equation, maximal functions, integral estimates, Sobolev spaces. 
Theorem B. Assume that $n \geq 1$ and $a \geq 2 s$ and $s>0$, and $b<2 s /(a-2 s)$. Assume also that

and that $f \in H_{s}$. Then

$$
\# A_{j} \lesssim 2^{b j} \text { for } j=1,2,3, \ldots
$$

$$
\lim _{k \rightarrow \infty} S_{t_{k}} f(x)=f(x)
$$

almost everywhere.

Theorem C. Assume that $n \geq 1$ and $a \geq 2 s$ and $s>0$, and that $\sum_{1}^{\infty} t_{k}^{\gamma}<\infty$, where $\gamma<2 s /(a-2 s)$. If also $f \in H_{s}$, then (2) holds almost everywhere.

Now let $E$ denote a bounded set in $\mathbf{R}^{n+1}$ and let $E_{0}$ denote the projection of $E$ onto the $t$-axis, i.e. $E_{0}=\left\{t\right.$; there extists $x \in \mathbf{R}^{n}$ such that $\left.(x, t) \in E\right\}$. For $a>0$ define an $a$-cube in $\mathbf{R}^{n+1}$ with side $r>0$ as an axis-parallell rectangular box with sidelength $r^{a}$ in the $t$-direction and with sidelength $r$ in the remaining directions $x_{1}, \ldots, x_{n}$. Thus an $a$-cube in $\mathbf{R}^{n+1}$ has volume $r^{n+a}$. For $r>0$ let $N_{E, a}(r)$ denote the minimal number $N$ of $a$-cubes $Q_{l}, l=1,2, \ldots, N$ of side $r$, such that $E \subset \bigcup_{1}^{N} Q_{l}$.

For $f \in \mathscr{S}$ we introduce the maximal function

$$
S_{E}^{*} f(x)=\sup _{(y, t) \in E}\left|S_{t} f(x+y)\right|, \quad x \in \mathbf{R}^{n} .
$$

We shall prove the following inequality.

Theorem 1. Assume that $n \geq 1$ and $a>0$ and $s>0$. If $f \in \mathscr{S}$, then one has

$$
\int_{\mathbf{R}^{n}}\left|S_{E}^{*} f(x)\right|^{2} d x \lesssim\left(\sum_{m=0}^{\infty} N_{E, a}\left(2^{-m}\right) 2^{-2 m s}\right)\|f\|_{H_{s}}^{2} .
$$

The following estimate follows directly.

Corollary 1. Assume $n \geq 1, a>0, s>0$ and $f \in \mathscr{S}$. If

$$
\sum_{m=0}^{\infty} N_{E, a}\left(2^{-m}\right) 2^{-2 m s}<\infty
$$

then

$$
\left\|S_{E}^{*} f\right\|_{2} \lesssim\|f\|_{H_{s}}
$$

We shall now describe a counter-example of of Sjögren and Sjölin [3]. Assume $a=2$ and let $\gamma: \mathbf{R}^{+} \rightarrow \mathbf{R}^{+}$be strictly increasing. Then there exist a function $f \in$ $H_{n / 2}$ and a continuous function $u(x, t)$ in $\{(x, t) ; t>0\}$ such that $u(x, t)=S_{t} f(x)$ and

$$
\limsup _{\substack{(y, t) \rightarrow(x, 0) \\|y-x|<\gamma(t), t>0}}|u(y, t)|=\infty
$$

for all $x \in \mathbf{R}^{n}$. This is generalized to a more general Schrödinger equation which contains the fractional Schrödinger equation $(a>1)$ by Johansson [2]. It follows that if $E=\{(y, t) ; 0<t<1,|y|<\gamma(t)\}$, then (3) does not hold for $s<n / 2$.

Now let $\Gamma:[0,1] \rightarrow\left(\mathbf{R}^{n}\right)$ and let $E$ be a subset of the graph of $\Gamma$ that is

$$
E \subset\{(\Gamma(t), t) ; 0 \leq t \leq 1\} .
$$

Assume that

$$
\left|\Gamma\left(t_{1}\right)-\Gamma\left(t_{2}\right)\right| \lesssim\left|t_{1}-t_{2}\right|^{\beta} \text { for } t_{1}, t_{2} \in E_{0}
$$


where $0<\beta$. Also set $a_{1}=1 / \beta$ and $a_{2}=\max \left(a, a_{1}\right)$. We shall first study the case when $E$ is the graph of $\Gamma$. Then (4) holds for any $\beta>1$ only if $\Gamma$ is constant. It follows from a result of Cho, Lee, and Vargas [1] that if $n=1, a=2, B$ is an interval of $\mathbf{R}$ and $E$ is the graph of $\Gamma$, then

$$
\left\|S_{E}^{*} f\right\|_{L^{2}(B)} \leq C_{B}\|f\|_{H_{s}} \text { if } s>\max (1 / 2-\beta, 1 / 4) \text {, and } 0<\beta \leq 1 .
$$

We have the following result.

Theorem 2. Assume $n \geq 1, a>0, f \in \mathscr{S}$ and that $E$ is the graph of $\Gamma$. Then (3) holds for $2 s>a_{2}$.

We shall then study the case when $E=\left\{\left(\Gamma\left(t_{k}\right), t_{k}\right) ; k=1,2,3 \ldots\right\}$, where the sequence $\left(t_{k}\right)_{1}^{\infty}$ satisfies (1). We have the following results.

Theorem 3. Assume $n \geq 1, a>0$, and $f \in \mathscr{S}$.

(i) In the case $2 s>a_{2}$, then (3) holds.

(ii) In the case $2 s=a_{2}$ assume that $\sum_{1}^{\infty} t_{k}^{\gamma}<\infty$ for some $\gamma>0$. Then (3) holds.

(iii) In the case $2 s<a_{2}$ assume that $\sum_{1}^{\infty} t_{k}^{\gamma}<\infty$ for some $\gamma$ satisfying $\gamma<$ $2 s /\left(a_{2}-2 s\right)$. Then (3) holds.

\section{Proof of Theorem 1}

If $y$ and $y_{0}$ belong to to $\mathbf{R}^{n}$ we write $y=\left(y_{1}, \ldots, y_{n}\right)$ and $y_{0}=\left(y_{0,1}, \ldots, y_{0, n}\right)$. We shall give the proof of Theorem 1 .

Proof. Assume $y_{0} \in \mathbf{R}^{n}, t_{0} \in \mathbf{R}, 0<r \leq 1$ and let

$$
E=\left\{(y, t) \in \mathbf{R}^{n+1} ; y_{0, j} \leq y_{j} \leq y_{0, j}+r \text { for } 1 \leq j \leq n \text {, and } t_{0} \leq t \leq t_{0}+r^{a}\right\} .
$$

We have

$$
S_{t} f(x+y)=c \int \mathrm{e}^{i \xi \cdot x} \mathrm{e}^{i \xi_{1} y_{1}} \ldots \mathrm{e}^{i \xi_{n} y_{n}} \mathrm{e}^{i t|\xi|^{a}} \hat{f}(\xi) d \xi
$$

where $c=(2 \pi)^{-n}$, and for $1 \leq j \leq n$ we write $\mathrm{e}^{i \xi_{j} y_{j}}=\Delta_{j}+\mathrm{e}^{i \xi_{j} y_{0, j}}$, where

$$
\Delta_{j}=\mathrm{e}^{i \xi_{j} y_{j}}-\mathrm{e}^{i \xi_{j} y_{0, j}} .
$$

We also write $\mathrm{e}^{i t|\xi|^{a}}=\Delta_{n+1}+\mathrm{e}^{i t_{0}|\xi|^{a}}$, where

$$
\Delta_{n+1}=\mathrm{e}^{i t|\xi|^{a}}-\mathrm{e}^{i t_{0}|\xi|^{a}} .
$$

Hence

$$
S_{t} f(x+y)=c \int \mathrm{e}^{i \xi \cdot x}\left(\Delta_{1}+\mathrm{e}^{i \xi_{1} y_{0,1}}\right) \ldots\left(\Delta_{n}+\mathrm{e}^{i \xi_{n} y_{0, n}}\right)\left(\Delta_{n+1}+\mathrm{e}^{i t_{0}|\xi|^{a}}\right) \hat{f}(\xi) d \xi
$$

and it follows that $S_{t} f(x+y)$ is the sum of integrals of the form

$$
c \int \mathrm{e}^{i \xi \cdot x}\left(\prod_{j \in D} \Delta_{j}\right)\left(\prod_{j \in B} \mathrm{e}^{i \xi_{j} y_{0, j}}\right) \Delta_{n+1} \hat{f}(\xi) d \xi,
$$

or

$$
c \int \mathrm{e}^{i \xi \cdot x}\left(\prod_{j \in D} \Delta_{j}\right)\left(\prod_{j \in B} \mathrm{e}^{i \xi_{j} y_{0, j}}\right) \mathrm{e}^{i t_{0}|\xi|^{a}} \hat{f}(\xi) d \xi .
$$

Here $D$ and $B$ ar disjoint subsets of $\{1,2,3, \ldots, n\}$ and $D \cup B=\{1,2,3, \ldots, n\}$. We denote the integrals in (5) by $S_{t}^{\prime} f(x, y)$ and shall describe how they can be estimated. The same argument works also for integrals in (6). 
For $j \in D$ we write

$$
\Delta_{j}=i \xi_{j} \int_{y_{0, j}}^{y_{j}} \mathrm{e}^{i \xi_{j} s_{j}} d s_{j}
$$

and we also write

$$
\Delta_{n+1}=i|\xi|^{a} \int_{t_{0}}^{t} \mathrm{e}^{i|\xi|^{a} s_{n+1}} d s_{n+1} .
$$

Assuming $D=\left\{k_{1}, k_{2}, \ldots, k_{p}\right\}$ we then have

$$
\begin{aligned}
S_{t}^{\prime} f(x, y)= & \int_{\mathbf{R}^{n}} \int_{y_{0, k_{1}}}^{y_{k_{1}}} \int_{y_{0, k_{2}}}^{y_{k_{2}}} \ldots \int_{y_{0, k_{p}}}^{y_{k_{p}}} \int_{t_{0}}^{t} \mathrm{e}^{i \xi \cdot x}\left(\prod_{j \in D} i \xi_{j} \mathrm{e}^{i \xi_{j} s_{j}}\right)\left(\prod_{j \in B} \mathrm{e}^{i \xi_{j} y_{0, j}}\right) \\
& \cdot i|\xi|^{a} \mathrm{e}^{i|\xi|^{a} s_{n+1}} \hat{f}(\xi) d s_{k_{1}} d s_{k_{2}} \ldots d s_{k_{p}} d s_{n+1} d \xi .
\end{aligned}
$$

Changing the order of integration one then obtains

$$
\begin{aligned}
\left|S_{t}^{\prime} f(x, y)\right| \leq & c \int_{y_{0, k_{1}}}^{y_{k_{1}}} \int_{y_{0, k_{2}}}^{y_{k_{2}}} \ldots \int_{y_{0, k_{p}}}^{y_{k_{p}}} \int_{t_{0}}^{t} \mid \int_{\mathbf{R}^{n}} \mathrm{e}^{i \xi \cdot x}\left(\prod_{j \in D} i \xi_{j} \mathrm{e}^{i \xi_{j} s_{j}}\right)\left(\prod_{j \in B} \mathrm{e}^{i \xi_{j} y_{0, j}}\right) \\
& \cdot i|\xi|^{a} \mathrm{e}^{i|\xi|^{a} s_{n+1}} \hat{f}(\xi) d \xi \mid d s_{k_{1}} d s_{k_{2}} \ldots d s_{k_{p}} d s_{n+1},
\end{aligned}
$$

or

$$
S_{t}^{\prime} f(x, y)=\int_{y_{0, k_{1}}}^{y_{k_{1}}} \ldots \int_{y_{0, k_{p}}}^{y_{k_{p}}} \int_{t_{0}}^{t} F_{D}\left(x ; s_{k_{1}}, \ldots, s_{k_{p}}, s_{n+1}\right) d s_{k_{1}} d s_{k_{2}} \ldots d s_{k_{p}} d s_{n+1},
$$

where

$$
F_{D}\left(x ; s_{k_{1}}, s_{k_{2}}, \ldots, s_{k_{p}}, s_{n+1}\right)=c \int_{\mathbf{R}^{n}} \mathrm{e}^{i \xi \cdot x}\left(\prod_{j \in D} i \xi_{j} \mathrm{e}^{i \xi_{j} s_{j}}\right)\left(\prod_{j \in B} \mathrm{e}^{i \xi_{j} y_{0, j}}\right) i|\xi|^{a} \mathrm{e}^{i|\xi|^{a} s_{n+1}} \hat{f}(\xi) d \xi .
$$

Then

$$
\begin{aligned}
& \sup _{(y, t) \in E}\left|S_{t}^{\prime} f(x, y)\right| \\
& \leq \int_{y_{0, k_{1}}}^{y_{0, k_{1}}+r} \ldots \int_{y_{0, k_{p}}}^{y_{0, k_{p}}+r} \int_{t_{0}}^{t_{0}+r^{a}}\left|F_{D}\left(x ; s_{k_{1}}, \ldots, s_{k_{p}}, s_{n+1}\right)\right| d s_{k_{1}} \ldots d s_{k_{p}} d s_{n+1} .
\end{aligned}
$$

Invoking Minkowski's inequality and Plancherel's formula we then obtain

$$
\begin{aligned}
& \left(\int_{\mathbf{R}^{n}} \sup _{(y, t) \in E}\left|S_{t}^{\prime} f(x, y)\right|^{2} d x\right)^{1 / 2} \\
& \leq \int_{y_{0, k_{1}}}^{y_{0, k_{1}}+r} \ldots \int_{y_{0, k_{p}}}^{y_{0, k_{p}}+r} \int_{t_{0}}^{t_{0}+r^{a}}\left\|F_{D}\left(\cdot ; s_{k_{1}}, \ldots, s_{k_{p}}, s_{n+1}\right)\right\|_{2} d s_{k_{1}} \ldots d s_{k_{p}} d s_{n+1} \\
& =c(2 \pi)^{n / 2} \int_{y_{0, k_{1}}}^{y_{0, k_{1}}+r} \ldots \int_{y_{0, k_{p}}}^{y_{0, k_{p}}+r} \int_{t_{0}}^{t_{0}+r^{a}}\left(\int_{\mathbf{R}^{n}}\left(\prod_{j \in D}\left|\xi_{j}\right|^{2}\right)|\xi|^{2 a}|\hat{f}(\xi)|^{2} d \xi\right)^{1 / 2} d s_{k_{1}} \\
& \ldots d s_{k_{p}} d s_{n+1} \leq r^{p} r^{a} A^{p} A^{a}\|f\|_{2}
\end{aligned}
$$

if $\left.f \in L^{2}\left(\mathbf{R}^{n}\right)\right)$ and supp $\hat{f} \subset B(0, A)$, where $A \geq 1$.

With similar arguments we get the estimate $r^{p} A^{p}\|f\|_{2}$ for the integrals in (6), and by summation of the all integrals of forms (5) or (6) we get

$$
\left\|\sup _{(y, t) \in E}\left|S_{t} f(x+y)\right|\right\|_{2} \leq(1+r A)^{n}\left(1+r^{a} A^{a}\right)\|f\|_{2} .
$$


Now let $E$ be a set in $\mathbf{R}^{n+1}$ with the property that $E \subset \bigcup_{1}^{N} Q_{l}$ where the sets $Q_{l}$ are $a$-cubes with side $r$ with $r A \leq 1$ of the type we have just considered. One has

$$
\sup _{(y, t) \in E}\left|S_{t} f(x+y)\right|^{2} \leq \sum_{1}^{N} \sup _{(y, t) \in Q_{l}}\left|S_{t} f(x+y)\right|^{2}
$$

and it follows from (7) that

$$
\int_{\mathbf{R}^{n}} \sup _{(y, t) \in E}\left|S_{t} f(x+y)\right|^{2} d x \leq 2^{2 n+2} N\|f\|_{2}^{2}
$$

if $r A \leq 1$ and supp $\hat{f} \subset B(0, A)$.

Now let $f \in \mathscr{S}$. We write $f=\sum_{k=0}^{\infty} f_{k}$, where the functions $f_{k}$ are defined in the following way. We set $\hat{f}_{0}(\xi)=\hat{f}(\xi)$ for $|\xi| \leq 1$ and $\hat{f}_{0}(\xi)=0$ for $|\xi|>1$. For $k \geq 1$ we let $\hat{f}_{k}(\xi)=\hat{f}(\xi)$ for $2^{k-1}<|\xi| \leq 2^{k}$ and $\hat{f}_{k}(\xi)=0$ otherwise.

Choosing real numbers $g_{k}>0, k=0,1,2, \ldots$, we have

$$
\begin{aligned}
\sup _{(y, t) \in E}\left|S_{t} f(x+y)\right| & \leq \sum_{k=0}^{\infty} \sup _{(y, t) \in E}\left|S_{t} f_{k}(x+y)\right| \\
& =\sum_{k=0}^{\infty} g_{k}^{-1 / 2} \sup _{(y, t) \in E}\left|S_{t} f_{k}(x+y)\right| g_{k}^{1 / 2} \\
& \leq\left(\sum_{k=0}^{\infty} g_{k}^{-1} \sup _{(y, t) \in E}\left|S_{t} f_{k}(x+y)\right|^{2}\right)^{1 / 2}\left(\sum_{k=0}^{\infty} g_{k}\right)^{1 / 2}
\end{aligned}
$$

and invoking inquality (8) we also have

$$
\begin{aligned}
& \int \sup _{(y, t) \in E}\left|S_{t} f(x+y)\right|^{2} d x \\
& \left.\leq\left.\left(\sum_{k=0}^{\infty} g_{k}^{-1} \int \sup _{(y, t) \in E} \mid S_{t} f_{k}(x+y)\right)\right|^{2} d x\right)\left(\sum_{k=0}^{\infty} g_{k}\right) \\
& \leq\left(\sum_{k=0}^{\infty} g_{k}\right)\left(\sum_{k=0}^{\infty} g_{k}^{-1} 2^{2 n+2} N_{E, a}\left(2^{-k}\right)\left\|f_{k}\right\|_{2}^{2}\right) .
\end{aligned}
$$

Chosing $g_{k}=N_{E, a}\left(2^{-k}\right) 2^{-2 k s}$ we conclude that

$$
\begin{aligned}
\int \sup _{(y, t) \in E}\left|S_{t} f(x+y)\right|^{2} d x & \leq 2^{2 n+2}\left(\sum_{k=0}^{\infty} N_{E, a}\left(2^{-k}\right) 2^{-2 k s}\right)\left(\sum_{k=0}^{\infty} 2^{2 k s}\left\|f_{k}\right\|_{2}^{2}\right) \\
& \lesssim\left(\sum_{k=0}^{\infty} N_{E, a}\left(2^{-k}\right) 2^{-2 k s}\right)\|f\|_{H_{s}}^{2},
\end{aligned}
$$

and the proof of Theorem 1 is complete.

\section{Proof of Theorems 2 and 3}

The following two lemmas follow easily from the definition of $N_{E, a}(r)$.

Lemma 1. Assume that $0<r \leq 1$ and $0<b<b_{1}$. Then

$$
N_{E, b}(r) \leq N_{E, b_{1}}(r),
$$


and

$$
N_{E, b_{1}}(r) \lesssim r^{b-b_{1}} N_{E, b}(r)
$$

Lemma 2. Assume that $E$ is a subset of the graph of $\Gamma$ satisfying (4) with $\beta>0$ and let $b \geq 1 / \beta$. Then

$$
N_{E, b}(r) \lesssim N_{E_{0}}\left(r^{b}\right) \text { for } 0<r \leq 1
$$

$\left(E_{0}, \Gamma, \beta\right.$ are defined and equation (4) is in Section 1).

We shall then give the proofs of Theorems 2 and 3.

Proof of Theorem 2. We have $a \leq a_{2}$. Invoking Lemma 1 and Lemma 2 we obtain

and

$$
N_{E, a}\left(2^{-m}\right) \leq N_{E, a_{2}}\left(2^{-m}\right) \lesssim N_{E_{0}}\left(2^{-m a_{2}}\right) \leq 2^{m a_{2}}+1
$$

$$
\sum_{m=0}^{\infty} N_{E, a}\left(2^{-m}\right) 2^{-2 m s}<\infty
$$

if $a_{2}-2 s<0$, i.e. $s>a_{2} / 2$. Using Corollary 1 we conclude that

$$
\left\|S_{E}^{*} f\right\|_{2} \lesssim\|f\|_{H_{s}}
$$

if $s>a_{2} / 2$.

We shall then prove Theorem 3.

Proof of Theorem 3. In the case $2 s>a_{2}$ we can use the same argument as in the proof of Theorem 2 to prove that (3) holds. Then assume $2 s \leq a_{2}$. We have $E_{0}=\left\{t_{k} ; k=1,2,3, \ldots\right\}$ and assuming $\sum_{1}^{\infty} t_{k}^{\gamma}<\infty$ one obtains $\# A_{j} \lesssim 2^{\gamma j}$. It then follows from Lemma 6 in [4] that $N_{E_{0}}\left(2^{-m}\right) \lesssim 2^{\gamma m /(\gamma+1)}$.

We have $a \leq a_{2}$. Applying Lemma 1 and Lemma 2 one obtains

$$
N_{E, a}\left(2^{-m}\right) \leq N_{E, a_{2}}\left(2^{-m}\right) \lesssim N_{E_{0}}\left(2^{-m a_{2}}\right) \lesssim 2^{a_{2} \gamma m /(\gamma+1)} .
$$

It follows that

$$
\sum_{m=0}^{\infty} N_{E, a}\left(2^{-m}\right) 2^{-2 m s}<\infty
$$

if $\gamma a_{2} /(\gamma+1)<2 s$, that is $a_{2} \gamma<2 s \gamma+2 s$. In the case $2 s=a_{2}$ this holds for every $\gamma>0$. In the case $2 s<a_{2}$ one has to assume $\gamma<2 s /\left(a_{2}-2 s\right)$. This completes the proof of Theorem 3 .

\section{References}

[1] Cho, C., S. Lee, and A. Vargas: Problems on pointwise convergence of solutions to the Schrödinger equation. - J. Fourier Anal. Appl. 18, 2012, 972-994.

[2] Johansson, K.: A counterexample on nontangential convergence for oscillatory integrals. Publ. Inst. Math. (Beograd) (N.S.) 87:101, 2010, 129-137.

[3] Sjögren, P., and P. Sjölin: Convergence properties for the time-dependent Schrödinger equuation. - Ann. Acad. Sci. Fenn. Math. 14, 1989, 13-25.

[4] Sjölin, P., and J.-O. Strömberg: Convergence of sequences of Schrödingers means. - J. Math. Anal. Appl. 483:1, 2020, 123580.

Received 11 June 2019 • Revised received 15 January 2020 • Accepted 25 May 2020 